\title{
Theoretical Model and Experimental Research on Friction and Torque Characteristics of Hydro-viscous Drive in Mixed Friction Stage
}

Hongwei Cui ${ }^{1} 2^{*} \mathbb{D}$, Qiliang Wang ${ }^{1}$, Zisheng Lian ${ }^{1,2}$ and Long Li ${ }^{1}$

\begin{abstract}
The hydro-viscous drive (HVD) has been widely used in fan transmission in vehicles, fans, and scraper conveyors for step-less speed regulation or soft starting. In the mixed friction stage, the contact, friction, and torque characteristics of friction pairs are very complex and change at any time. The characteristics of the frictional and hydrodynamic lubrication states were studied in order to calculate and predict the friction and torque characteristics of the friction pairs in the mixed friction stage. The fluid torque was calculated by applying the average shear stress model and the load-carrying capacity of asperity was determined on the basis of the fractal contact theory. In addition, the contact friction coefficient of the friction pairs was taken into consideration and measured by using the MM1000-III friction and wear testing machine. The asperity friction torque and total torque in the mixed friction stage were obtained and finally, the test rig for the torque characteristics was set up. The results show that the contribution to the total torque is shared by the oil film and the asperity friction. The friction coefficient decreases sharply at first and then increases with a change in the relative rotational speed, following the Stribeck curve closely, and the contact frictional coefficient slowly decreases with increase in the pressure between the friction pairs. The torque between the friction pairs is provided by the asperity friction, and the torque due to the oil film reduces to zero. When the thickness of the oil film is small, a major contribution to the total torque is due to the asperity friction. The total torque also increases with the decrease in the film thickness ratio. Therefore, by theoretical analysis and experimental verification, the torque of the friction pairs in the mixed friction stage can be accurately calculated using the average shear stress model and asperity friction torque model.
\end{abstract}

Keywords: Hydro-viscous drive (HVD), Mixed friction stage, Torque characteristics, Contact friction coefficient

\section{Introduction}

The hydro-viscous drive (HVD) which is widely used in fans, pumps, belt conveyors, and scraper conveyors is a kind of torque and power transmission device based on oil film shearing and friction for step-less speed regulation, soft starting, and overload protection [1]. The HVD mainly consists of a series of friction plates (FP) and separator discs (SD) and the torque is generated by the relative rotation of these. The output speed can be changed by changing the thickness of the oil film, which can be

\footnotetext{
*Correspondence: cuihongwei@tyut.edu.cn

${ }^{1}$ College of Mechanical and Vehicle Engineering, Taiyuan University of Technology, Taiyuan 030024, China

Full list of author information is available at the end of the article
}

accomplished by a clutch piston that is acted by control oil. There are three stages of speed regulation: (i) fluid transmission by shear stress, (ii) mixed friction, where the surface asperities come into contact as the thickness of the fluid film decreases, and (iii) rough contact stage until the relative velocity becomes zero $[2,3]$.

In mixed friction, the load-carrying capacity and torque are shared by the oil film and asperity contact. However, the ratio, distribution area, and contact status of the oil film and asperity contact are very complicated. In addition, the load-carrying, torque, and frictional coefficient constantly change with the thickness of the oil film and the working conditions [4].

A lot of research has been conducted on the torque characteristics while using a wet clutch. A novel method 
considering the clutch friction coefficient for estimating driveline torques for a vehicle with a dual-clutch transmission was proposed [5]. A fractal model of elastic-plastic contact between three-dimensional rough surfaces was built using the modified two-variable WeierstrassMandelbrot function [6]. Zhao et al. developed a model of plane contacts in mixed lubrication, and pin-on-disc tests were carried out. The real contact area ratio, load sharing ratio, and friction coefficient were investigated [7, 8]. Based on the fluid lubrication and Hertz contact theory, the characteristics of contact and torque in the case of a wet clutch were obtained, and the wear model was established by using the fractal theory [9]. Based on the existing formulas, the torque stability of the friction pairs in the mixed friction stage was studied [10]. The influencing factors of the friction characteristics of the paperbased wet clutch were analyzed considering the influence of surface topography of the friction pairs [11]. Experimental research on contact area and the effect on friction torque of paper-based friction pairs was carried out [12].

Therefore, based on the calculation model of loadcarrying capacity and torque in case of the wet clutch, the friction and torque characteristics of the HVD were determined [13, 14]. Besides, Berger [15] believed that the viscous torque of the friction pairs is small, and the friction torque is mainly provided by the asperity contact. The contact model of a rough surface was set up in the mixed friction stage of the HVD by outlining the asperity contact properties [16]. An experimental research on friction characteristics of friction pairs in the HVD was conducted [17]. Although the structure of the HVD and wet clutch are similar, the working conditions are completely different. The engagement time of the wet clutch is very short, but the HVD is in a relative sliding and mixed friction state for a long time for stable speed regulation. Therefore, the friction and torque characteristics are different in the working process of the HVD and wet clutch.

In fact, the contact and friction characteristics in the mixed friction stage are greatly time-varying and difficult to predict accurately. The changes in the friction coefficient play an important role in the calculation of friction torque. The friction coefficient model was analyzed and established on the basis of the sliding speed, the pressure, and the surface temperature of the clutch plates and the calculation model of the friction torque of a dry clutch was constructed [18]. The friction coefficient, local friction torque, and total output torque were analyzed under the effects of thermal buckling of $\mathrm{Cu}$-based friction pairs in a wet multidisk clutch [19]. The frictional torque of the wet clutch was calculated considering the effects of surface roughness, permeability, elastic modulus of the frictional material, lubricant viscosity, and temperature by using the analytical model and experimental measurement [20]. The friction characteristics of the wet clutch were tested using the SAE \#2 test rig, and the conclusion was that the friction coefficient is affected by relative sliding speed, pressure, and temperature [21]. The jitter and stick-slip of the wet clutch during engagement which is caused by the change of friction torque and friction coefficient was analyzed [22, 23].

It is obvious that the friction torque of the HVD in the mixed friction stage cannot be calculated by the traditional model, especially as the friction coefficient is not measured and predicted accurately. Therefore, the friction and torque characteristics of friction pairs in the mixed friction stage were studied. The fluid torque was calculated by applying the average shear stress model. The contact friction coefficient was taken into consideration and measured using the MM1000-III friction and wear testing machine. The changes in the asperity friction torque and total torque were obtained. The calculated results provide a theoretical basis for research of frictional and torque characteristics of friction pairs in the HVD.

\section{State Analysis of Mixed Friction Stage}

The friction plate and separator disc are shown in Figure 1 . The material of the $\mathrm{SD}$ is $32 \mathrm{CrMnSiA}$. The core of the FP is $65 \mathrm{Mn}$, and the copper based powder metallurgy friction material is sintered on both sides. The friction material has the advantages of good speed regulation performance, high temperature resistance, and high reliability. Therefore, the friction materials are widely used in HVD, wet clutch, friction brake, and other mechanical transmission. The double arcs oil grooves are machined on the surface of the FP. $\psi$ is defined as the coefficient of effective area of the friction pairs, which is the ratio of the non-grooves area to the total area.

Figure 2 is the schematic diagram of friction pairs in the mixed friction stage. The FP and SD are composed of different roughness. The area between the inner and outer diameters is the nominal contact area $A_{\mathrm{a}}$, which is made up of asperity contact and oil film shear zones.

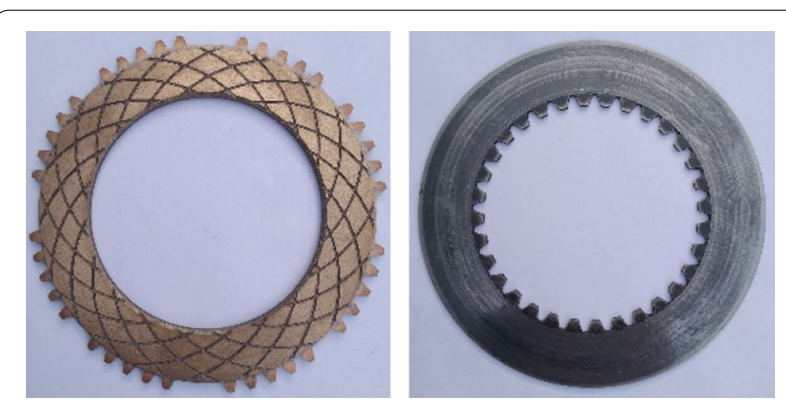

Figure 1 Friction pairs of HVD 


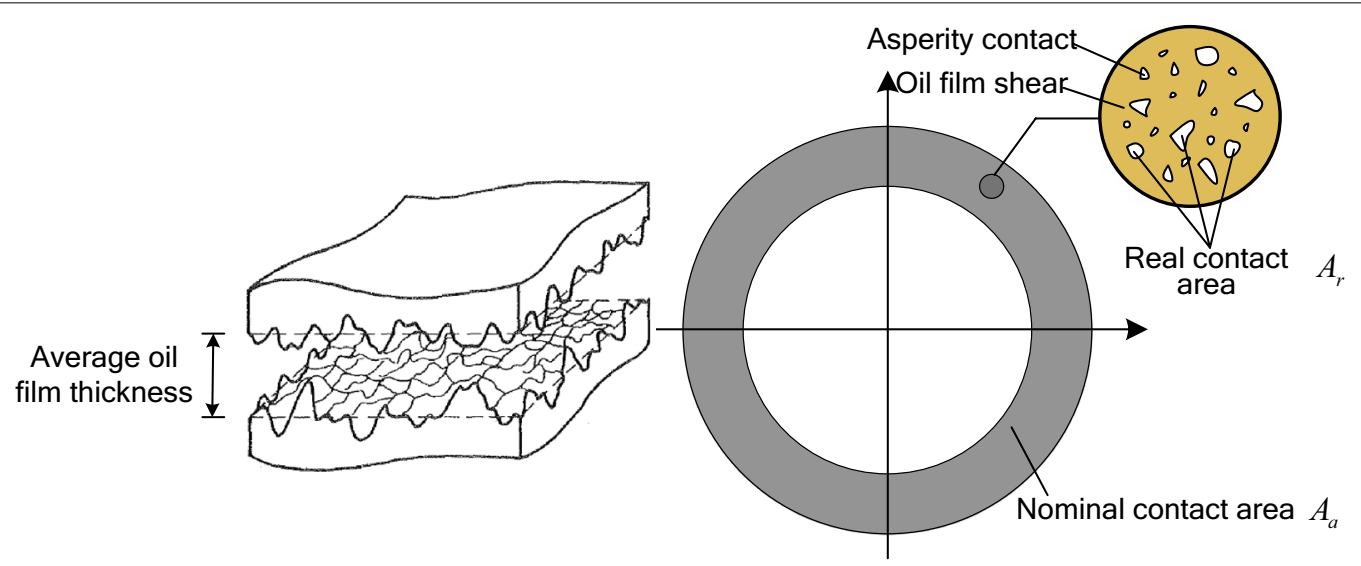

Figure 2 Schematic diagram of mixed friction stage

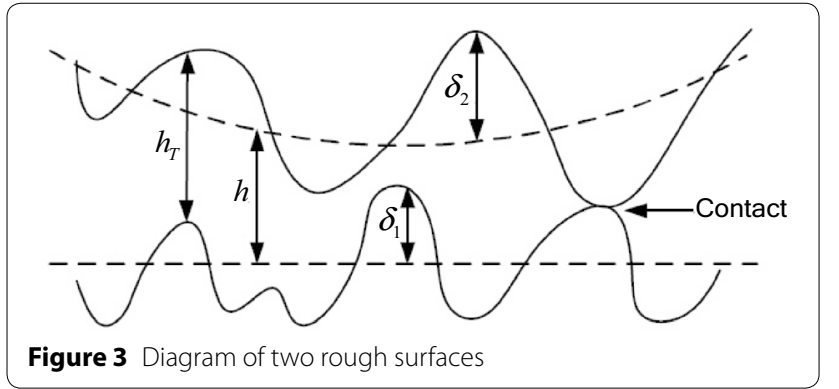

The asperity contact area, which is only a part of the nominal contact area, is the real contact area $A_{\mathrm{r}}$. The total external load $W_{\mathrm{f}}$, provided by the control oil pressure, is shared by the oil film $W_{\mathrm{h}}$ and asperity contact $W_{\mathrm{a}}$, i.e., $W_{\mathrm{f}}=W_{\mathrm{h}}+W_{\mathrm{a}}$, and the total torque $T_{\mathrm{f}}$ is made up of the fluid torque $T_{\mathrm{h}}$ and asperity friction torque $T_{\mathrm{a}}$, i.e., $T_{\mathrm{f}}=T_{\mathrm{h}}+T_{\mathrm{a}}$.

\subsection{Film Thickness Equation}

The nominal film thickness $h$ is the distance between the centerlines of two rough surface profiles, as shown in Figure 3, but the actual film thickness $h_{T}$ is a random variable obtained from the probability of surface roughness. $\delta_{1}$ and $\delta_{2}$ are the heights of the asperity peaks. $\bar{h}_{T}$ is the average actual film thickness given by [24]
In general, the effect of roughness on lubrication can be ascertained by the film thickness ratio. When $\lambda<3$, the asperity contact will become evident, and then the friction pairs are said to be in the mixed friction stage.

\subsection{Contact Area Ratio}

The contact area ratio $k_{\mathrm{a}}$ is defined as the ratio of the real contact area to nominal contact area, which changes with thickness of the oil film.

Assuming that the asperity peaks on the surface satisfy the Gaussian distribution with zero average value, Greenwood and Tripp calculated the actual contact area ratio based on the G-W model as [5]

$$
k_{a}=\frac{\pi^{2}\left(\eta_{p} R_{p} \sigma\right)^{2}}{2}\left[\left(1+\lambda^{2}\right) \cdot \operatorname{erfc}\left(\frac{\lambda}{\sqrt{2}}\right)-\sqrt{\frac{2}{\pi}} \lambda e^{-\frac{\lambda^{2}}{2}}\right],
$$

where $\eta_{p}$ is the peak density of the rough surface, and $R_{p}$ is the radius of curvature of the asperity peak. $\operatorname{erfc}(\cdot)$ is the Gaussian residual error function given by $\operatorname{erfc}(x)=2 / \sqrt{\pi} \int_{x}^{\infty} e^{-\eta^{2}} \mathrm{~d} \eta$.

The actual contact area of the friction pairs changing with the film thickness ratio is given as

$$
A_{r}=A_{a} k_{a} .
$$

$$
\bar{h}_{T}= \begin{cases}h, & \lambda \geq 3, \\ \frac{3 \sigma}{256}\left[35+z\left(128+z\left(140+z^{2}\left(-70+z^{2}\left(28-5 z^{2}\right)\right)\right)\right)\right], & \lambda<3,\end{cases}
$$

where $z=\lambda / 3$ and $\sigma$ is the root mean square roughness. $\lambda$ is the film thickness ratio given by

$$
\lambda=\frac{h}{\sigma}=\frac{h}{\sqrt{\sigma_{1}^{2}+\sigma_{2}^{2}}} .
$$

\section{Fluid Torque Model}

Based on the Newton's law for internal friction and the average flow model, the average shear stress model, in which the influence of surface roughness of the friction pairs on fluid torque is considered, is defined as follows [25]: 


$$
\bar{\tau}=\frac{\mu\left(\omega_{1}-\omega_{2}\right) r}{\bar{h}_{T}}\left(\phi_{f}-\phi_{f s}\right),
$$

where $\bar{\tau}$ is the average shear stress, $\phi_{f}$ and $\phi_{f s}$ are the shear stress factors, $\mu$ is the dynamic viscosity of the fluid, $\omega_{1}$ is the angular velocity of the FP, and $\omega_{2}$ is the angular velocity of the $\mathrm{SD}$.

In Eq. (5), the shear stress will be infinite when the thickness of the oil film approaches zero, and when it is where $R_{1}$ and $R_{2}$ are the inner and outer radii of the friction pairs respectively.

\section{Asperity Friction Torque Model}

\subsection{Load-Carrying Capacity of Asperity}

Based on the fractal contact theory, the M-B fractal contact model of the friction pairs was established, and the load-carrying capacity of asperity in the mixed friction stage was obtained [26].

If $a_{l}>a_{c}$, the total load is given by

$$
W_{a}^{*}= \begin{cases}\frac{4 \sqrt{\pi}}{3} E G^{*(D-1)} g_{1}(D) \Psi^{(D-2)^{2} / 4} A_{r}^{* D / 2} \times\left[\left(\frac{2-D}{D} \Psi^{-(2-D) / 2} A_{r}^{*}\right)^{(3-2 D) / 2}-a_{c}^{*(3-2 D) / 2}\right]+H g_{2}(D) \Psi^{(D-2)^{2} / 4} A_{r}^{* D / 2} a_{c}^{*(2-D) / 2}, & D \neq 1.5, \\ 3^{-3 / 4} \sqrt{\pi} E G^{* 1 / 2} \Psi^{1 / 16} A_{r}^{* 3 / 4} \ln \left[\frac{A_{r}^{*}}{3 \Psi^{1 / 4} a_{c}^{*}}\right]+3^{1 / 4} H \Psi^{1 / 16} A_{r}^{* 3 / 4} a_{c}^{* 1 / 4}, & D=1.5,\end{cases}
$$

close to the value of the molecular diameter, the Newton's law for internal friction is not valid. Therefore, a quantity $\varepsilon$ needs to be defined, and the shear stress is assumed to be zero when the thickness of the oil film is less than this value $\varepsilon$.

The shear stress factor $\phi_{f}$ is the average of the sliding speed of friction surfaces and given by where $E$ is the equivalent elastic modulus, $D$ is the fractal dimension, $G$ is the scale factor, and $H$ is the hardness of softer materials.

If $a_{l}<a_{c}$, the total load is given by

$$
W_{a}^{*}=H A_{r}^{*} .
$$

$a_{l}$ is the maximum area of the contact point

$$
\phi_{f}=\left\{\begin{array}{cc}
\frac{35}{32} z\left\{\begin{array}{r}
\left(1-z^{2}\right)^{3} \ln \frac{z+1}{\varepsilon^{*}}+\frac{1}{60}[-55+z(132+z(345 \\
+z(-160+z(-405+z(60+147 z)))))]
\end{array}\right\}, \quad \lambda \leq 3, \\
\frac{35}{32} z\left\{\left(1-z^{2}\right)^{3} \ln \frac{z+1}{z-1}+\frac{z}{15}\left[66+z^{2}\left(30 z^{2}-80\right)\right]\right\}, & \lambda>3,
\end{array}\right.
$$

where $\varepsilon^{*}=0.00333$.

The expression for the shear stress factor $\phi_{f s}$ is as follows:

$$
\phi_{f s}=V_{r 1} \Phi_{f_{s}}(\lambda)-V_{r 2} \Phi_{f_{s}}(\lambda)
$$

where $V_{r 1}=\left(\sigma_{1} / \sigma\right)^{2}, V_{r 2}=\left(\sigma_{2} / \sigma\right)^{2}$,

$$
\Phi_{f s}= \begin{cases}A_{3} \lambda^{\alpha_{4}} e^{-\alpha_{5} \lambda+\alpha_{6} \lambda^{2}}, & \lambda \leq 7 \\ 0, & \lambda>7\end{cases}
$$

where $A_{3}, \alpha_{4}, \alpha_{5}$ and $\alpha_{6}$ are the corresponding coefficients.

In the mixed friction stage, because the depth of the oil groove is much larger than the thickness of the oil film, the shear torque of the oil groove area is much smaller than that of the non-groove area. Therefore, the influence of the flow in the groove area and the shape of the groove on the torque due to the oil film are neglected.

Therefore, the fluid torque of the friction pairs in the average shear stress model in the mixed friction stage can be expressed as

$$
\begin{aligned}
T_{h} & =\left(1-k_{a}\right) \psi \int_{R_{1}}^{R_{2}} \int_{0}^{2 \pi} \frac{\mu\left(\omega_{1}-\omega_{2}\right) r^{3}}{\bar{h}_{T}}\left(\phi_{f}-\phi_{f s}\right) \mathrm{d} \theta \mathrm{d} r \\
& =\left(1-k_{a}\right) \psi \frac{\pi \mu\left(\omega_{1}-\omega_{2}\right)\left(R_{2}^{4}-R_{1}^{4}\right)}{2 \bar{h}_{T}}\left(\phi_{f}-\phi_{f_{s}}\right),
\end{aligned}
$$

$$
A_{r}=\frac{D}{2-D} \Psi^{(2-D) / 2} a_{l}
$$

$a_{c}$ is the critical contact area given by

$$
a_{c}=G^{2}\left(\frac{\pi E^{2}}{225 \sigma_{y}^{2}}\right)^{1 /(D-1)}
$$

where $\sigma_{y}$ is the yield strength of softer materials.

Each parameter is expressed as follows:

$$
\begin{aligned}
& \left\{\begin{array}{l}
g_{1}(D)=\frac{D}{(3-2 D)}\left(\frac{2-D}{D}\right)^{D / 2}, \\
g_{2}(D)=\left(\frac{D}{2-D}\right)^{(2-D) / 2},
\end{array}\right. \\
& W_{a}^{*}=\frac{W_{a}}{A_{a}} ; \quad G^{*}=\frac{G}{\sqrt{A_{a}}} ; \quad A_{r}^{*}=\frac{A_{r}}{A_{a}}=k_{a} ; \quad a_{c}^{*}=\frac{a_{c}}{A_{a}} .
\end{aligned}
$$

The nominal contact area is:

$$
A_{a}=\psi \pi\left(R_{2}^{2}-R_{1}^{2}\right)
$$

$\Psi$ can be obtained by the following equation: 


$$
\frac{\Psi^{(2-D) / 2}-\left(1+\Psi^{-D / 2}\right)^{-(2-D) / D}}{(2-D) / D}=1 .
$$

\subsection{Contact Friction Coefficient}

The contact friction coefficient which is defined for the calculation of asperity friction torque is only caused by the asperity contact and friction in the mixed friction stage. It is a part of the total friction coefficient but is affected by the oil film and is different from dry friction. The change rule of the contact friction coefficient can be obtained by measuring the torque characteristics of friction pairs by the test method.

\subsubsection{MM1000-III Friction and Wear Testing Machine}

MM1000-III friction and wear testing machine is suitable for the testing the brake and transmission performance of friction pairs in the fields of aerospace, rail trains, automobiles, and construction machinery. The dry friction braking performance test and wet inertial transmission performance test of friction pairs made of metallic, nonmetallic, and composite materials can be carried out.

The testing machine mainly consists of a mechanical system, power system, control system, and data test system. The structural diagram is shown in Figure 4.

\subsubsection{Test Principle and Scheme}

The FP is located on the main shaft and can rotate but the $\mathrm{SD}$ is fixed in a circumferential direction. The working oil flows from the inner to the outer diameter of the friction pairs through the clearance and oil grooves. The type of relative friction and oil supply methods are consistent with the friction pairs in the HVD.

Firstly, an inertia load is selected and the FP rotates with the main shaft after the main motor is started. After reaching the set stable speed, the main shaft disengages from the main motor by using the clutch. At the same time, a pressure is provided for the friction pairs through the cylinder. The FP and SD slide against each other until the rotation of the FP becomes zero.

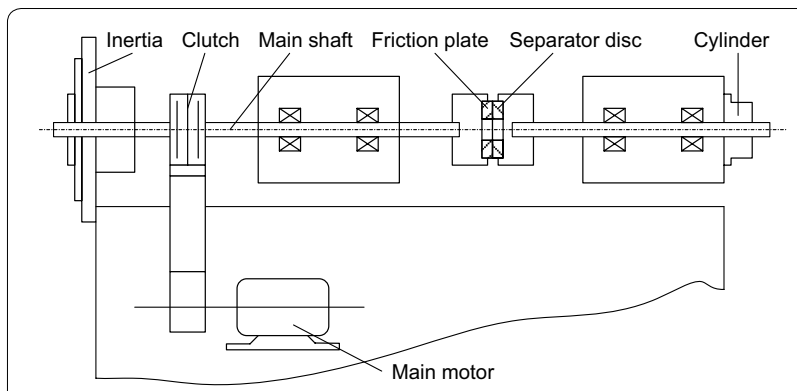

Figure 4 Structure of MM1000-III friction and wear testing machine
The friction torque can be measured by the torque sensor and the speed of the FP by the speed sensor. The pressure between the friction pairs can be obtained by the pressure of the cylinder and effective area of the friction pairs. The temperature of the friction pairs can be determined by a temperature sensor that is installed on the SD. The dynamic frictional coefficient can be calculated at different relative sliding velocities.

The rotating speed of the main shaft was $4200 \mathrm{r} / \mathrm{min}$. The inertia load was $0.6 \mathrm{~kg} \cdot \mathrm{m}^{2}$, and the braking tests were conducted when the pressure of the friction pairs was varied from $0.1 \mathrm{MPa}$ to $1 \mathrm{MPa}$.

\subsubsection{Analysis of Test Results}

Generally speaking, the frictional coefficient is always affected by the temperature, pressure between the FP and SD, properties of the working oil, and relative sliding speed. All other factors being the same, the variation of frictional coefficient with relative speed under different pressures was determined by using the friction and wear testing machine, as shown in Figure 5.

The variation in the frictional coefficient with the relative speed was first analyzed. As shown in Figure 5, in the low speed area where the relative speed is less than $600 \mathrm{r} / \mathrm{min}$, the frictional coefficient decreases sharply, from around 0.14, with increase in the relative speed. The large frictional coefficient-relative speed gradient is caused by the difference between the dynamic and static frictional coefficients. At a certain value of the relative speed, the frictional coefficient increases, but the gradient is small. The reason is that a partial oil film exists between the friction pairs in the mixed friction stage, and the viscous shear stress of the oil film increases with the relative speed. In addition, as the pressure of the friction pairs increases, the area of the oil film decreases, hence, the effect of the shear stress decreases gradually.

The test results of the frictional coefficient are very similar to the Stribeck curve, which is shown in Figure 6 [27]. The friction coefficient decreases sharply and then increases slowly with the increase of relative sliding velocity.

If the effect of the shear stress on the frictional coefficient is detracted, the contact frictional coefficient can be obtained [27]. The graph between the contact frictional coefficient and relative speed is approximately parallel to the horizontal axis in the high-speed area, as shown in Figure 7.

The large negative gradient in the frictional coefficientrelative speed curve indicates that it is an unstable speed regulation stage in the low speed area. Therefore, the stable speed regulation stage in the high-speed area is considered where the contact frictional coefficient does not change with variation in the relative speed. 


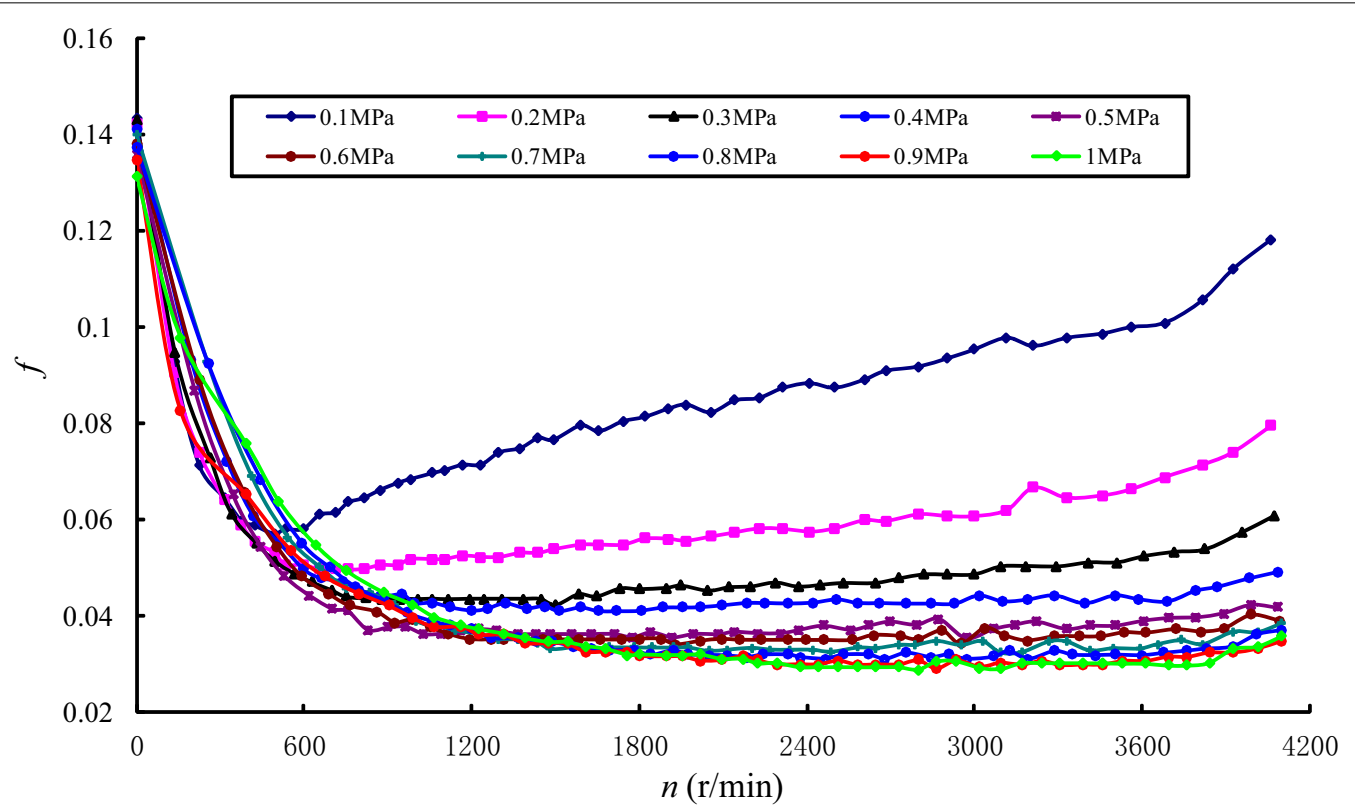

Figure 5 Variation of frictional coefficient with relative speed under different pressure

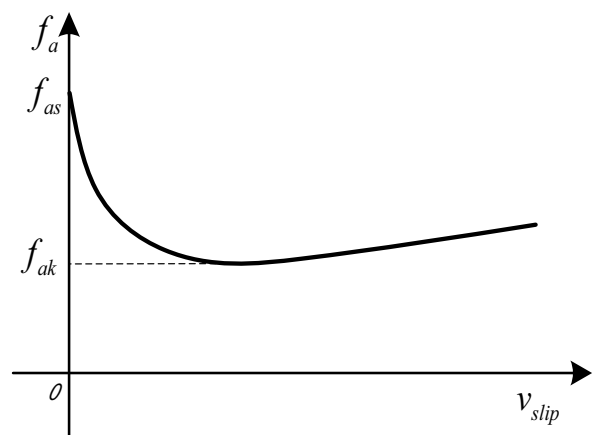

Figure 6 Stribeck curve $\left(f_{a k}\right.$ is the dynamic friction coefficient, $f_{a s}$ is the maximum static friction coefficient, $v_{\text {slip }}$ is the relative sliding velocity)

In order to determine the relationship between the contact frictional coefficient and the film thickness ratio, the influence of pressure on the contact frictional coefficient should be considered. The values of the contact frictional coefficient at different pressures were extracted from the test data as shown in Table 1. After polynomial fitting, the relationship between the contact frictional coefficient and pressure was obtained as given in Equation (18), which is shown in Figure 8. It can be concluded that the contact frictional coefficient gradually decreases with pressure and can be used to calculate the asperity friction torque.

$$
f_{a}=0.073-0.1557 p_{a}+0.233 p_{a}^{2}-0.1595 p_{a}^{3}+0.0379 p_{a}^{4},
$$

where $f_{a}$ is the contact frictional coefficient and $p_{a}$ is the pressure.

The contact frictional coefficients are measured repeatedly by the testing machine for the same conditions of initial rotating speed, inertia, and pressure. The temperature of the friction pairs, obtained approximately from the maximum value of the temperature sensor on the $\mathrm{SD}$, gradually rises due to the power loss of friction. The relationship between the contact frictional coefficient and temperature at a pressure of $0.6 \mathrm{MPa}$ is shown in Figure 9. It can be seen that with the increase of temperature, the contact frictional coefficient remains basically unchanged. The main reason is that the copper based powder metallurgy friction material has better thermal stability of friction coefficient within this temperature range.

\subsection{Asperity Friction Torque}

After the load-carrying capacity of asperity and the contact frictional coefficient under different film thickness ratio were determined, the asperity friction torque can be calculated as follows:

$$
T_{a}=\frac{W_{a} f_{a}\left(R_{1}+R_{2}\right)}{2} .
$$

\section{Experimental Research}

A test rig was built to analyze the torque characteristics of the HVD in the mixed friction stage and verify the theoretical model. The components are shown in Figure 10. 


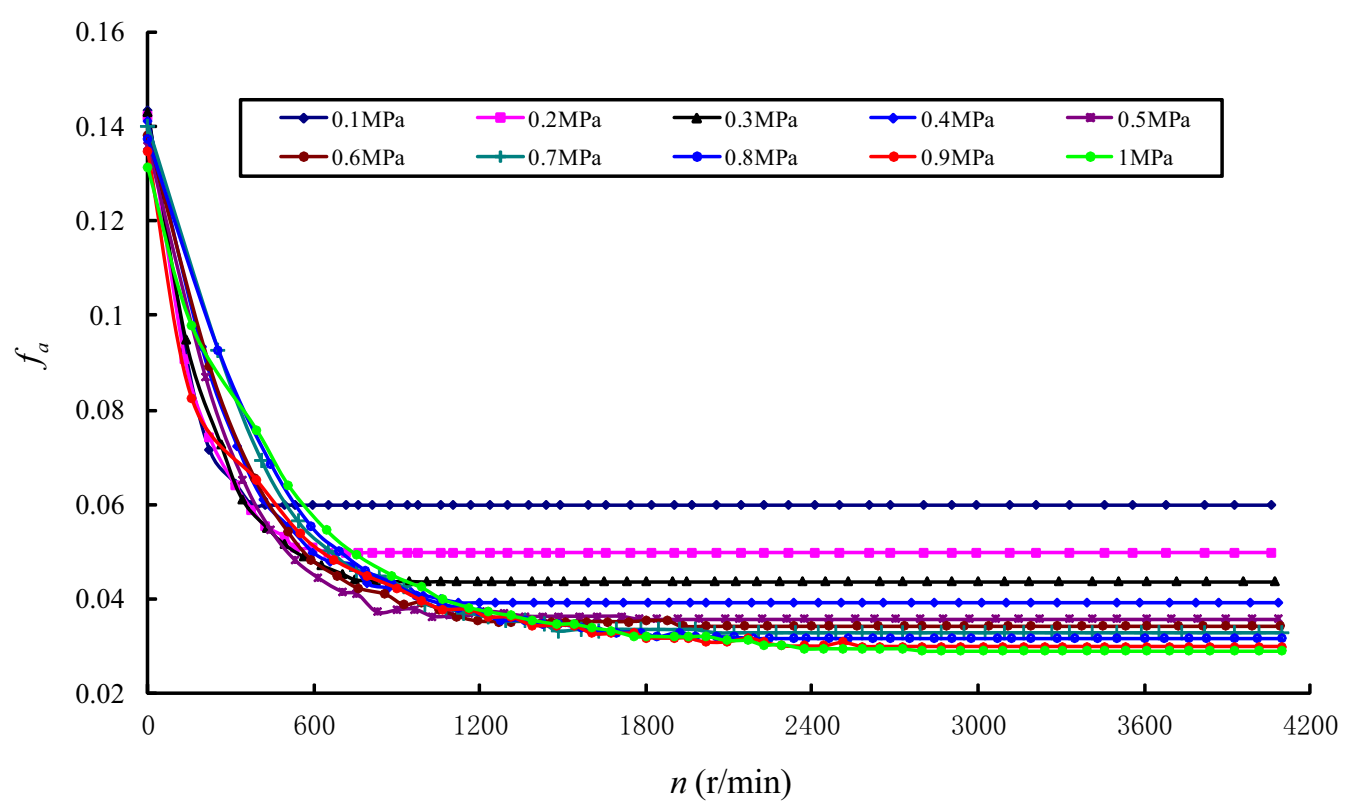

Figure 7 Variation of contact frictional coefficient

Table 1 Contact frictional coefficient at different pressures

\begin{tabular}{llllll}
\hline$p_{\mathrm{a}}(\mathrm{MPa})$ & 0.1 & 0.2 & 0.3 & 0.4 & 0.5 \\
$f_{\mathrm{a}}$ & 0.06 & 0.05 & 0.044 & 0.039 & 0.036 \\
$p_{\mathrm{a}}(\mathrm{MPa})$ & 0.6 & 0.7 & 0.8 & 0.9 & 1 \\
$f_{\mathrm{a}}$ & 0.034 & 0.033 & 0.0315 & 0.03 & 0.029 \\
\hline
\end{tabular}

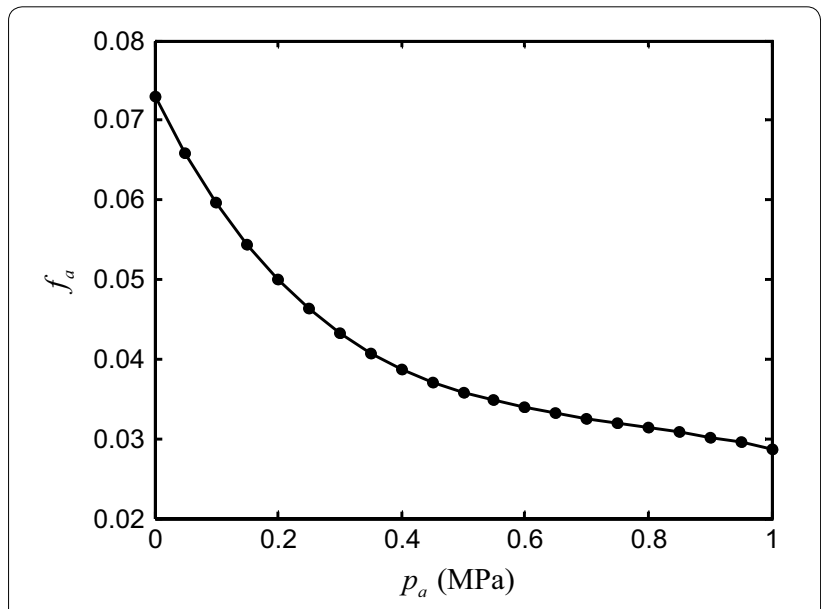

Figure 8 Variation of contact frictional coefficient with pressure

The test rig consists of a mechanical system, an operational control system, a hydraulic system, and a test system. The HVD is driven by a motor, and the load is

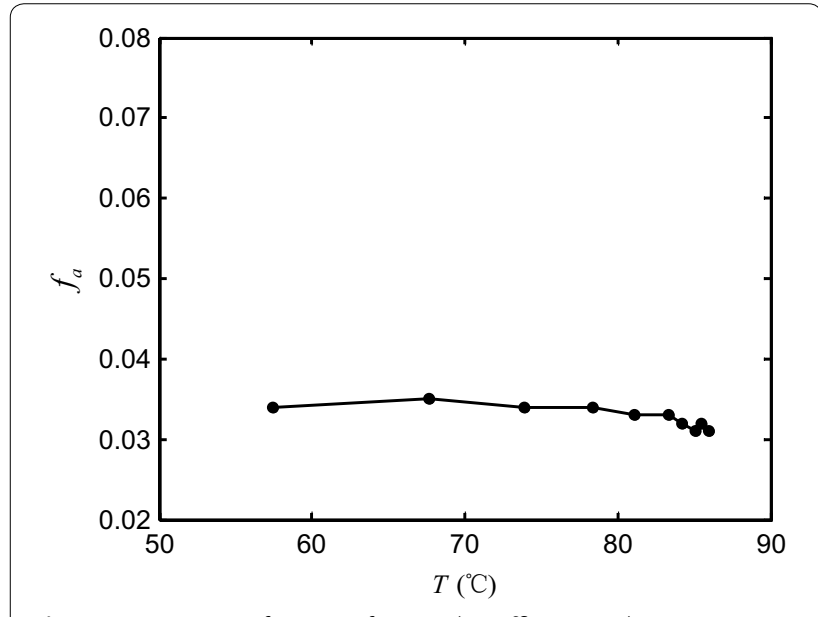

Figure 9 Variation of contact frictional coefficient with temperature

provided by an eddy current dynamometer. Inside the HVD, a thrust bearing is placed in the axial direction between the plate and piston as shown in Figure 11. The cylinder and piston do not rotate in the tangential direction, therefore, it is convenient to install the displacement transducer. After the displacement of the piston is accurately measured, the thickness of the oil film and film thickness ratio can be calculated.

The hydraulic system consists of the channels of the working oil and controlling oil. The working oil is supplied to the gap between the friction pairs and plays the role of coolant. The gaps between the friction pairs can 


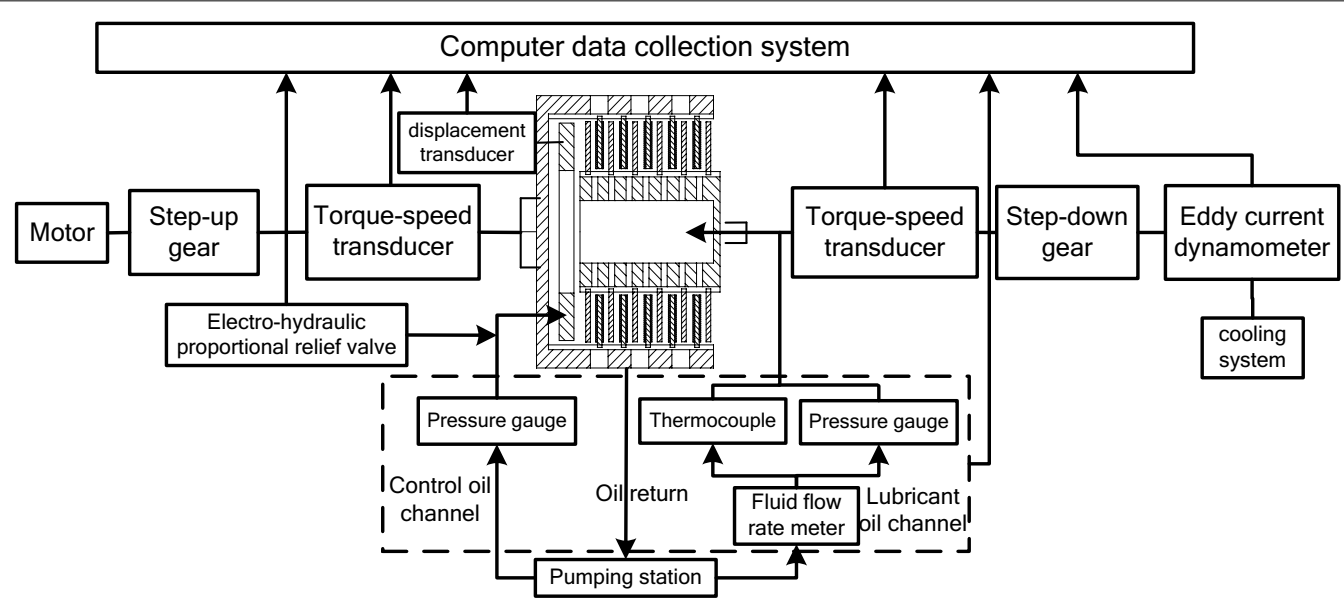

Figure $\mathbf{1 0}$ Components of the test rig

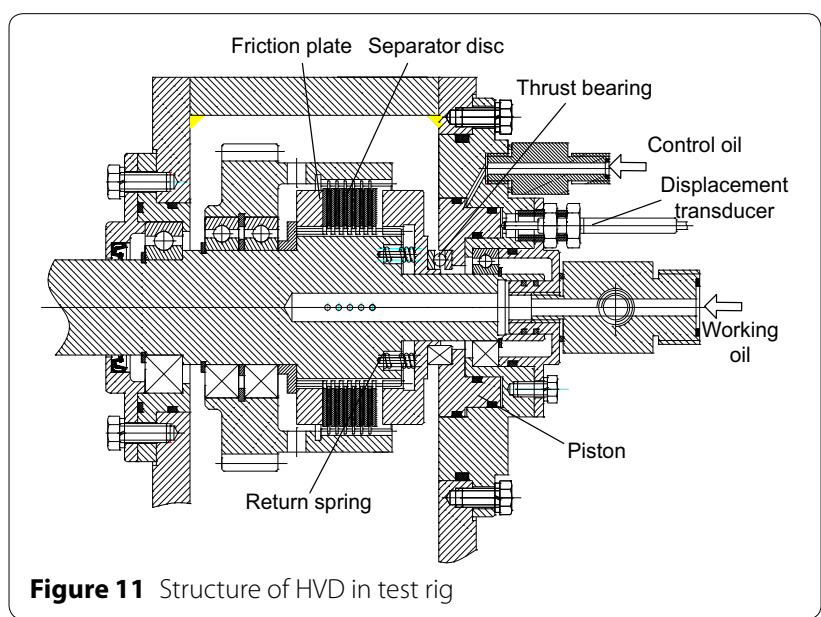

be adjusted by the electro-hydraulic proportional relief valve to control the oil pressure.

During the test, the output of the HVD is in brake state, and the input rotational speed is constant. The pressure of the control oil is increased gradually to change the thickness of the oil film, so that the mixed friction stage can be examined and the variation of the torque of the friction pairs with the thickness of the oil film can be measured.

The test system includes a torque-speed transducer for measuring the speed of rotation and torque of the input and output. The temperature and pressure of the lubricant oil can be measured by a thermocouple and pressure gauge, respectively. The displacement transducer is used to measure the thickness of the oil film. The loadcarrying capacity of the friction pairs can be calculated by measuring the pressure of the control oil.
Table 2 Parameters of structure, material, and working conditions

\begin{tabular}{ll}
\hline Parameter & Value \\
\hline Inner radius $R_{1}(\mathrm{~mm})$ & 43 \\
Outer radius $R_{2}(\mathrm{~mm})$ & 61.5 \\
Root mean square of roughness of FP $\sigma_{1}(\mu \mathrm{m})$ & 5.975 \\
Root mean square of roughness of SD $\sigma_{2}(\mu \mathrm{m})$ & 2.85 \\
Fractal dimension of FP D & 1.54 \\
Scale factor of FP $G$ & $1.584 \times 10^{-11}$ \\
Coefficient of effective area $\psi$ & 0.78 \\
Angular velocity of $\mathrm{FP} \omega_{1}(\mathrm{rad} / \mathrm{s})$ & 104.7 \\
Angular velocity of SD $\omega_{2}(\mathrm{rad} / \mathrm{s})$ & 0 \\
Dynamic viscosity of oil $\mu(\mathrm{Pa} \cdot \mathrm{s})$ & 0.055 \\
Peak density of the rough surface $\eta_{p}\left(\mathrm{~m}^{-2}\right)$ & $8.49 \times 10^{7}$ \\
Curvature radius of asperity peak $R_{p}(\mathrm{~m})$ & $8 \times 10^{-4}$ \\
Equivalent elastic modulus of $\mathrm{FP} E(\mathrm{GPa})$ & 2.176 \\
Compressive strength of $\mathrm{FP}(\mathrm{MPa})$ & 110 \\
Hardness of $\mathrm{FP} H\left(\mathrm{~N} / \mathrm{mm}^{2}\right)$ & 50
\end{tabular}

\section{Results and Discussion}

Using the formulas of the fluid torque and asperity friction torque models, the relation between the torque of the friction pairs and film thickness ratio was obtained. The parameters of the structure, material, and working conditions are shown in Table 2.

\subsection{Fluid Torque}

The variation in the thickness of the oil film is shown in Figure 12. Both the average of the actual thickness of the oil film $\bar{h}_{T}$ and the nominal thickness of the oil film $\mathrm{h}$ increase with the film thickness ratio $\lambda$. When $\lambda>1.5$, they are basically the same. However, when $0<\lambda<1.5$, $\bar{h}_{T}$ is slightly greater than $h$. When $\lambda=0, \bar{h}_{T}$ is not zero. 


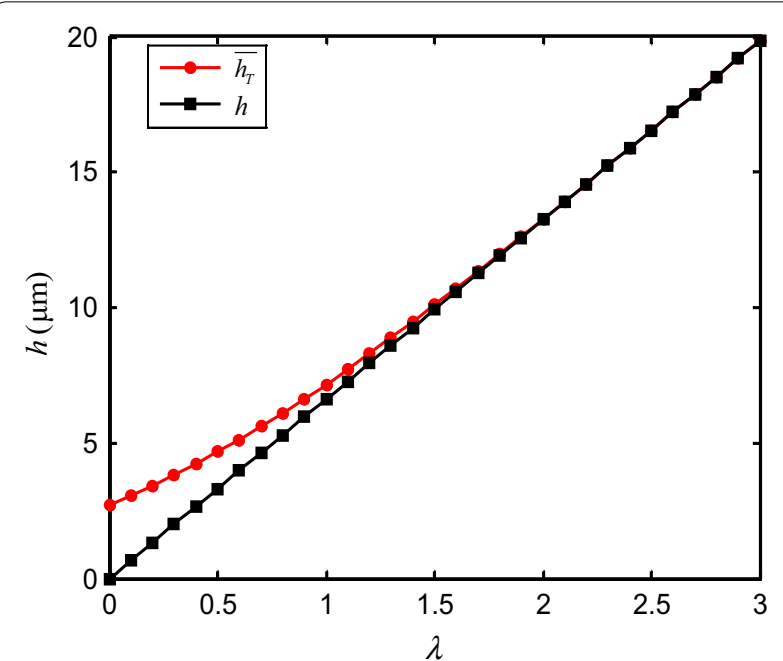

Figure 12 Variation of actual oil film thickness and nominal film thickness with film thickness ratio

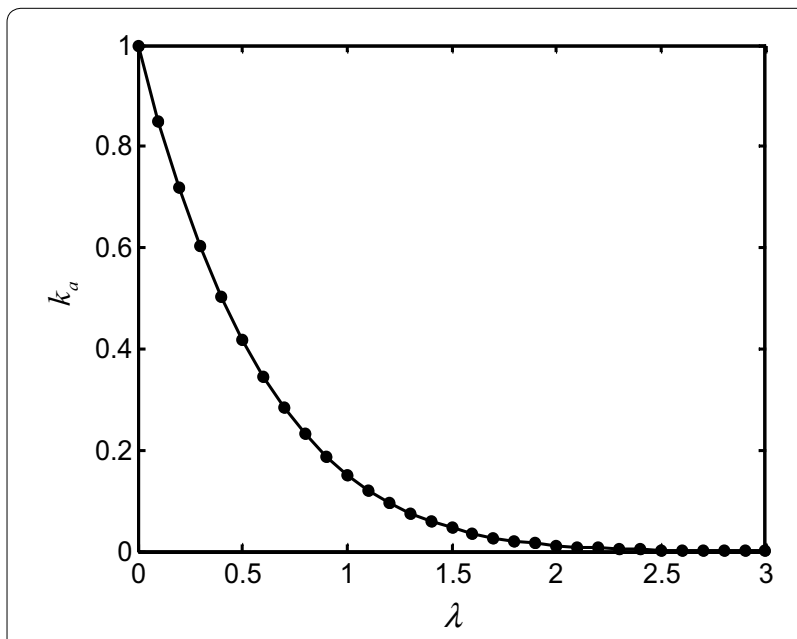

Figure 13 Variation of contact area ratio with film thickness ratio

Therefore, the calculation of the shear stress and fluid torque by using Newton's internal friction law is valid.

The variation of the contact area ratio with the film thickness ratio is shown in Figure 13. When $0<\lambda<1$, the contact area ratio decreases sharply with the film thickness ratio. At this time, there is a large real contact area between the friction pairs, and the asperity contact plays a major role. When $1<\lambda<3$, the contact area ratio decreases slowly and when $\lambda>2$, the contact area is almost zero. At this juncture, the load between the friction pairs is mainly provided by the oil film.

In the mixed friction stage, the variation of the fluid torque with film thickness ratio is shown in Figure 14. When $0<\lambda<2$, the fluid torque increases sharply from zero with the film thickness ratio. The main reason is

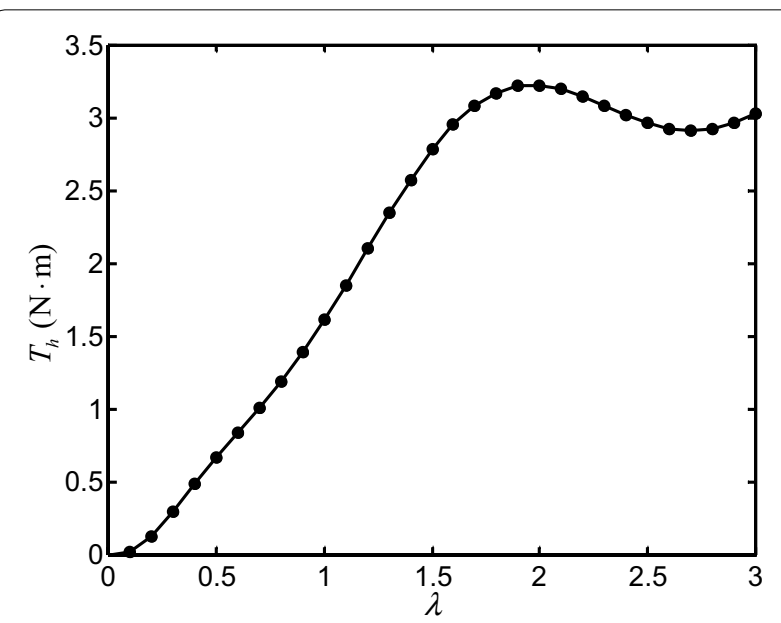

Figure 14 Variation of oil film shear torque with film thickness ratio

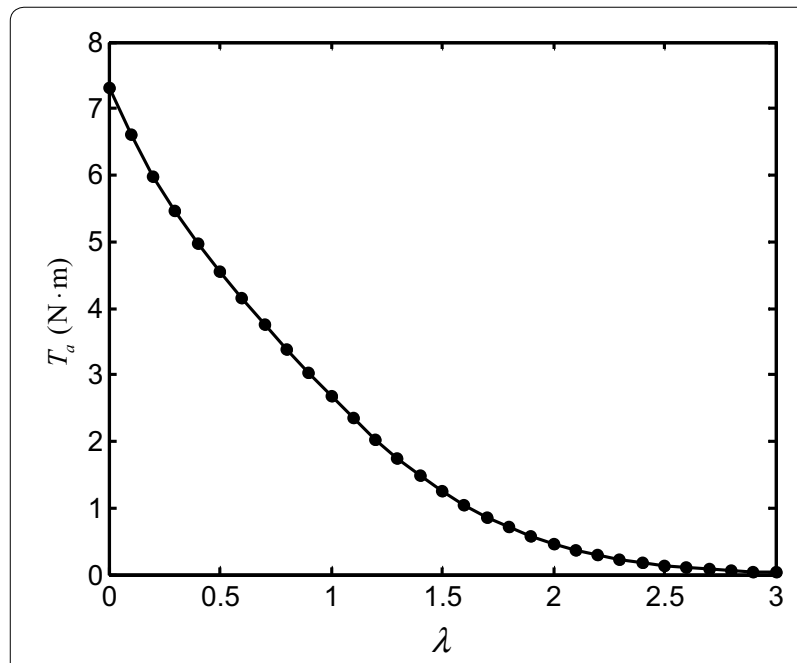

Figure 15 Variation of asperity friction torque with film thickness ratio

that the real contact area of asperity decreases and the area of the oil film increases gradually. At the same time, the total shear stress factor of the fluid torque increases sharply, too. When $2<\lambda<3$, the fluid torque decreases slowly from the maximum value. This is because the contact area ratio is basically unchanged with respect to the film thickness ratio (Figure 13), but the total shear stress factor gradually decreases.

\subsection{Asperity Friction Torque}

Figure 15 shows the variation of the asperity friction torque with film thickness ratio. In the mixed friction stage, the torque, at first, increases slowly from zero and then increases sharply until it reaches a maximum value as the film thickness ratio decreases. The reason is that 
the real contact area plays a more important role in the asperity friction torque. A comparison with Figure 13 shows that with the decrease in thickness of the oil film, the rate of increase of the asperity friction torque is lesser than that of the contact area ratio. This is because the contact frictional coefficient gradually decreases with decrease in the film thickness ratio and pressure.

\subsection{Total Torque}

The total torque can be calculated by adding the fluid torque and asperity friction torque. Figure 16(a) shows the variation of the asperity friction torque, fluid torque, and the total torque with the film thickness ratio in the mixed friction stage. Figure 16(b) shows the result in the test conditions. A comparison shows that the experimental results and theoretical values are consistent.

When $1.2<\lambda<3$, as the real contact area of the friction pairs is small, the fluid torque accounts for a large

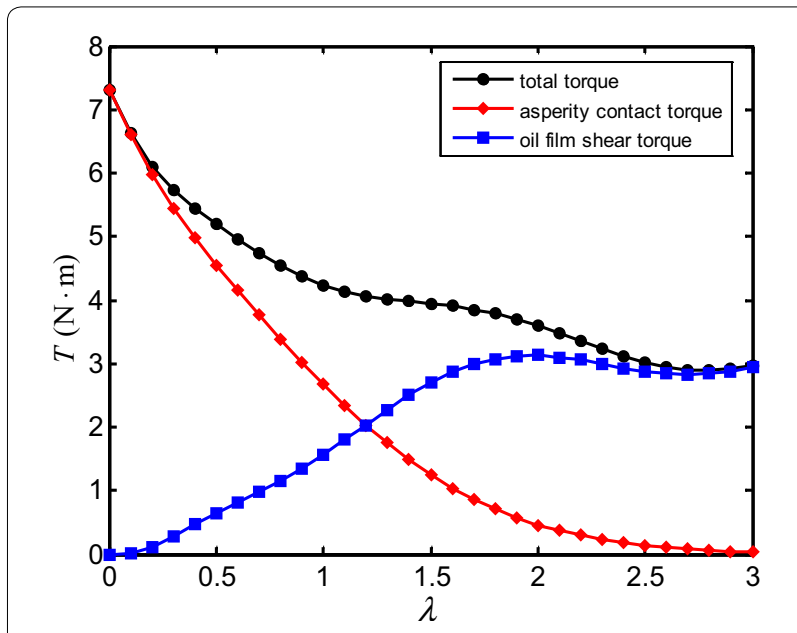

a theoretical torque

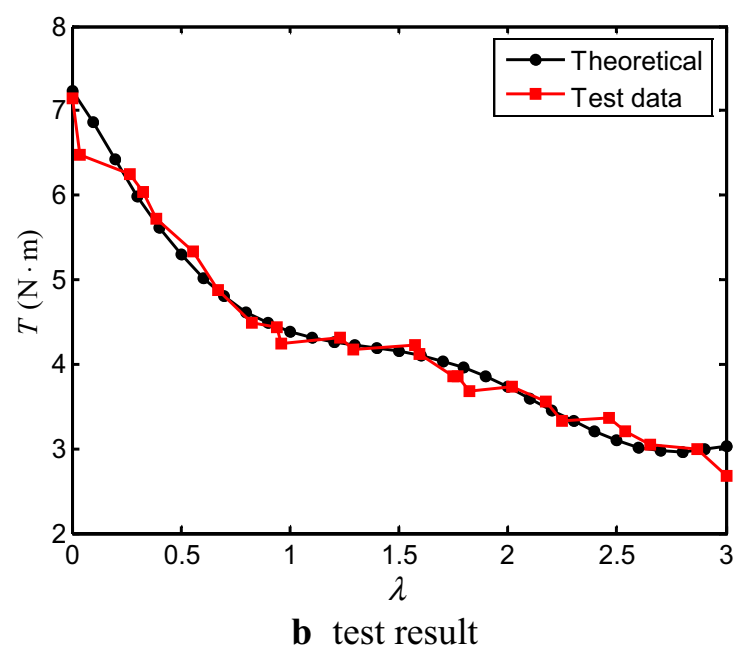

Figure 16 Variation of torque with film thickness ratio proportion of the total torque, and the total torque increases as the film thickness ratio decreases. When $0<\lambda<1.2$, with the decrease in the film thickness ratio, the real asperity contact area increases. Therefore, the contribution of the asperity friction torque is more than the fluid torque, and hence, the total torque increases sharply.

\section{Conclusions}

The calculation of the torque characteristics of friction pairs in the mixed friction stage was carried out using the theoretical model as well as experimental research. The conclusions are as follows:

1. The friction coefficient of the friction pairs in the mixed friction stage was determined by using the MM1000-III friction and wear testing machine. The friction coefficient decreased sharply at first and then increased with a change in the relative rotational speed, closely following the Stribeck curve. At the same time, by processing the experimental data, the contact frictional coefficient was obtained to calculate the asperity friction torque, which gradually decreased with pressure between the friction pairs.

2. In the mixed friction stage, the contribution to the total torque was shared by the oil film shear and asperity friction. The contact area ratio increased with a decrease in the film thickness ratio. The torque between the friction pairs was provided by the asperity friction, and the torque due to the oil film reduced to zero. When the thickness of the oil film was small, the asperity friction torque played a more important role in contributing to the total torque, which increased with the decrease in the film thickness ratio.

3. The experimental results and theoretical values were consistent. Therefore, in the mixed friction stage, the torque of the friction pairs could be calculated by using the average shear stress model and asperity friction torque model in which the contact friction coefficient was taken into consideration and measured.

\section{Authors' Contributions}

ZL was in charge of the whole trial; $\mathrm{HC}$ wrote the manuscript; $\mathrm{QW}$ and $\mathrm{LL}$ assisted with sampling and laboratory analyses. All authors read and approved the final manuscript.

\section{Authors' Information}

Hongwei Cui, born in 1986, is currently an Associate Professor at Shanxi Key Laboratory of Fully Mechanized Coal Mining Equipment, Taiyuan University of Technology, China. He received his PhD degree from Beijing Institute of Technology, China, in 2014. His research interests include fluid power transmission and control, hydro-viscous drive and coal mine machinery. 
Qiliang Wang, born in 1991, is currently a PhD candidate at Taiyuan University of Technology, China. He received his bachelor degree from Taiyuan University of Technology, China, in 2013.

Zisheng Lian, born in 1959, is currently a Professor at Shanxi Key Laboratory of Fully Mechanized Coal Mining Equipment, Taiyuan University of Technology, China. He received his PhD degree from China University of Mining \& Technology, Beijing, China, in 1995. His research interests include fluid power transmission and control and coal mine machinery.

Long Li, born in 1988, is currently a PhD candidate at Taiyuan University of Technology, China. He received his master degree from Lanzhou University of Technology, China, in 2010.

\section{Competing Interests}

The authors declare that they have no competing interests.

\section{Funding}

Supported by National Natural Science Foundation of China (Grant Nos. 51805351, U1810123).

\section{Author Details}

${ }^{1}$ College of Mechanical and Vehicle Engineering, Taiyuan University of Technology, Taiyuan 030024, China. ${ }^{2}$ Shanxi Key Laboratory of Fully Mechanized Coal Mining Equipment, Taiyuan University of Technology, Taiyuan 030024, China.

Received: 30 July 2018 Revised: 29 April 2019 Accepted: 17 September 2019

Published online: 01 October 2019

\section{References}

[1] FW Xie. Influence law of temperature field and deformed interface on hydro-viscous drive characteristics. Xuzhou: China University of Mining and Technology, 2010. (in Chinese)

[2] HW Cui, S Y Yao, Q D Yan, et al. Mathematical model and experiment validation of fluid torque by shear stress under influence of fluid temperature in hydro-viscous clutch. Chinese Journal of Mechanical Engineering, 2014, 27(1): 32-40

[3] HW Cui, Z S Lian, Y Y Deng, et al. The research on characteristics of flow field and shear torque of oil film for hydro-viscous drive. JFPS International Journal of Fluid Power System, 2017, 10(2): 9-17.

[4] Q J Wang, Y W Chung. Encyclopedia of Tribology. Boston: Springer, 2013.

[5] S Kim, J J Oh, S B Choi. Driveline torque estimations for a ground vehicle with dual-clutch transmission. IEEE Transactions on Vehicular Technology, 2018, 67(3): 1977-1989.

[6] R F Yu, W Chen. Fractal modeling of elastic-plastic contact between three-dimensional rough surfaces. Industrial Lubrication and Tribology, 2018, 70(2): 290-300.

[7] E H Zhao, B Ma, HY Li. Numerical and experimental studies on tribological behaviors of cu-based friction pairs from hydrodynamic to boundary lubrication. Tribology Transactions, 2017, https://doi.org/10.1080/10402 004.2017.1323145.

[8] E H Zhao. Research on friction and wear characteristics of sliding cu-based friction pairs in wet multi-disc clutches. Beijing: Beijing Institute of Technology, 2017. (in Chinese)

[9] Z G Zhang. Study on several working characteristics of wet clutch. Hangzhou: Zhejiang University, 2010. (in Chinese)
[10] T M Cameron, T D Mark, M Tracy. Clutch parameter effects on torque and friction stability. SAE Technical Paper, 2011, https://doi. org/10.4271/2011-01-0722.

[11] B H Li. Influence and mechanism of surface topography on friction characteristics of wet clutches. Beijing: China Academy of Machinery Science and Technology, 2006: 76-87. (in Chinese)

[12] Y Kimura, C Otani. Contact and wear of paper-based friction materials for oil-immersed clutches-wear model for composite materials. Tribology International, 2005 (38): 943-950.

[13] Q R Meng. Study on hydro-viscous drive speed regulating start and control technology. Xuzhou: China University of Mining and Technology, 2008. (in Chinese)

[14] Y Hong. Study behavior of speeding wet clutch and fuzzy controller. Shanghai: Shanghai University, 2004. (in Chinese)

[15] E J Berger, F Sadeghi, C M Krousgrill. Torque transmission characteristics of automatic transmission suet clutches: experimental results and numerical comparison. STLE Tribology Transaction, 1997, 40: 539-548.

[16] J Z Cui. Research on stability of the soft-start and the mechanism of power transmission in hydro-viscous drive. Zhenjiang: Jiangsu University, 2015. (in (hinese)

[17] L Y Guo, M G Du. Friction and thermal load performance of a viscous clutch. Tribology, 2011, 31 (4): 323-327.

[18] X H Lin, J Q Xi, S Q Hao. The calculation model of the friction torque on a dry clutch. Proceedings of the Institution of Mechanical Engineers, Part D: Journal of Automobile Engineering, 2017: 1-10.

[19] E H Zhao, B Ma, HY Li. The tribological characteristics of cu-based friction pairs in a wet multidisk clutch under nonuniform contact. Journal of Tribology, 2018, 140: 011401 (1-9).

[20] J Cho, Y Lee, W Kim, et al. Wet single clutch engagement behaviors in the dual-clutch transmission system. International Journal of Automotive Technology, 2018, 19(3): 463-472.

[21] Y Yang, R Lam, T Fujii. Prediction of torque response during the engagement of wet friction clutch, SAE Technical Paper, 1998, No. 981097.

[22] A Crowther, N Zhang. Torsional finite elements and nonlinear numerical modelling in vehicle powertrain dynamics. Journal of Sound and Vibration, 2005, 284(3-5): 825-849.

[23] A Crowther, N Zhang. Analysis and simulation of clutch engagement judder and stick-slip in automotive powertrain systems. Proceedings of the Institution of Mechanical Engineering, Part D: Journal of Automobile Engineering, 2004, 218, 1427-1446.

[24] N Patir, H S Cheng. An average flow model for determining effects of three-dimensional roughness on partial hydrodynamic lubrication. Journal of Lubrication Technology, 1978, 100: 12-17.

[25] N Patir, H S Cheng. Application of average flow model to lubrication between rough sliding surfaces. Journal of Lubrication Technology, 1979, 101: 220-230

[26] HW Cui. Research on the torque characteristic of friction pairs in hydroviscous clutch. Beijing: Beijing Institute of Technology, 2014. (in Chinese)

[27] W J Ding. Self-excited vibration. Beijing: Tsinghua University Press, 2009.

\section{Submit your manuscript to a SpringerOpen ${ }^{\circ}$ journal and benefit from:}

- Convenient online submission

- Rigorous peer review

- Open access: articles freely available online

- High visibility within the field

Retaining the copyright to your article

Submit your next manuscript at springeropen.com 